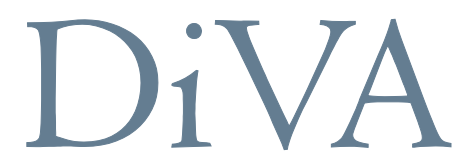

http://uu.diva-portal.org

This is an author produced version of a paper published in Thin Solid Films. This paper has been peer-reviewed but does not include the final publisher proofcorrections or journal pagination.

Citation for the published paper:

Valyukh, I. et. al.

"Optical properties of thin films of mixed $\mathrm{Ni}-\mathrm{W}$ oxide made by reactive DC magnetron sputtering"

Thin Solid Films, 2011, Vol. 519, Issue 9: 2914-2918

URL: http://dx.doi.org/10.1016/j.tsf.2010.11.089

Access to the published version may require subscription.

Published with permission from: Elsevier 


\title{
Optical Properties of Thin Films of Mixed Ni-W Oxide Made by Reactive DC Magnetron Sputtering
}

\author{
I. Valyukh ${ }^{1}$, S.V. Green ${ }^{2}$, C.G. Granqvist ${ }^{2}$, G.A. Niklasson ${ }^{2}$, S. Valyukh ${ }^{1}$, H. Arwin ${ }^{1}$ \\ ${ }^{1}$ Laboratory of Applied Optics, Department of Physics, Chemistry and Biology, \\ Linköping University, SE-58183 Linköping, Sweden \\ ${ }^{2}$ Department of Engineering Sciences, The Ångström Laboratory, Uppsala University, \\ P.O. Box 534, SE-75121 Uppsala, Sweden
}

Contact author: I. Valyukh, e-mail: iryva@ifm.liu.se

Fax: +46 13 137568; Tel.: +4613281000

\begin{abstract}
Thin films of $\mathrm{Ni}_{x} \mathrm{~W}_{1-x}$ oxides with $x=0.05,0.19,0.43$ and 0.90 were studied. Films with thicknesses in the range $125-250 \mathrm{~nm}$ were deposited on silicon wafers at room temperature by reactive $\mathrm{DC}$ magnetron co-sputtering from targets of $\mathrm{Ni}$ and $\mathrm{W}$. The films were characterized with X-ray diffraction (XRD), scanning electron microscopy (SEM), and spectroscopic ellipsometry (SE). XRD spectra and SEM micrographs showed that all films were amorphous and possessed a columnar structure. The ellipsometric angles $\Psi$ and $\Delta$ of as-deposited films were measured by a rotating analyzer ellipsometer in the UV-visible-near infrared range (0.63-6.18 eV) and by an infrared Fourier transform rotating compensator ellipsometer in the $500-5200 \mathrm{~cm}^{-1}$ wavenumber range. SE measurements were performed at angles of incidence of from $50^{\circ}$ to $70^{\circ}$. Parametric models were used to extract thicknesses of the thin films and overlayers of $\mathrm{Ni}_{x} \mathrm{~W}_{1-x}$ oxide at different compositions, band gaps and optical constants. Features in the optical spectra of the $\mathrm{Ni}_{x} \mathrm{~W}_{1-x}$ oxides were compared with previous data on tungsten oxide, nickel oxide and nickel tungstate.
\end{abstract}

Key words: tungsten oxide, nickel oxide, ellipsometry.

\section{Introduction}


Amorphous tungsten oxide $\left(\mathrm{WO}_{3}\right)$ and polycrystalline nickel oxide $(\mathrm{NiO})$ are among the most widely used electrochromic (EC) materials [1-3]. They are of great technological importance for the development of coatings with tunable optical properties and have found application in many devices such as "smart windows" for energy efficient buildings, information displays, and rear view mirrors for automobiles.

Intensive research efforts are currently focused on finding new EC materials with improved optical and electro-optical characteristics. One interesting possibility in these endeavours involves the incorporation of transition metal atoms into $\mathrm{WO}_{3}$ and $\mathrm{NiO}$. With this in mind, we have recently embarked on a detailed study of the $\mathrm{Ni}_{x} \mathrm{~W}_{1-x}$ oxide system [4-6] and demonstrated that superior optical properties can be obtained for intermediate compositions $[6,7]$.

In previous work we have determined the optical constants of amorphous $\mathrm{WO}_{3}$ and polycrystalline $\mathrm{NiO}$ thin films [5]. In the present work, we extend these studies to thin films of $\mathrm{Ni}_{x} \mathrm{~W}_{1-x}$ oxides with different compositions prepared by co-sputtering. Specifically we use spectroscopic ellipsometry (SE) to characterize these thin films and to investigate the evolution of optical constants with increasing Ni content.

The optical constants of the materials under study depend both on composition of the $\mathrm{Ni}_{x} \mathrm{~W}_{1-x}$ oxide and on the film structure. The composition of the films was characterized by the parameter $x$, that can be accurately determined by Rutherford Backscattering Spectroscopy (RBS) or X-ray Photoelectron Spectroscopy (XPS) [6]. Previous work using XPS and Raman spectroscopy (RS) has indicated a complex structure, including the presence of amorphous $\mathrm{WO}_{3}$ as well as nanosized $\mathrm{NiO}$ and nickel tungstate $\left(\mathrm{NiWO}_{4}\right)$ phases in the films. Particularly, XPS and Raman spectroscopy showed clear evidence that $\mathrm{Ni}_{x} \mathrm{~W}_{l-x} \mathrm{O}_{y}$ thin films are composed of $\mathrm{WO}_{y}$, (with y close to 3 ) and $\mathrm{NiWO}_{4}$ phases for $x<0.5$, and that the main constituent is $\mathrm{NiWO}_{4}$ for $x=0.5$ [6]. In films with $x>0.5$, XPS gave clear evidence for $\mathrm{NiO}$ and the presence of reduced tungsten ions $\left(\mathrm{W}^{5+}\right)$, as well as weak indications of hydrated phases and $\mathrm{NiWO}_{4}$ [6]. Studies of the film structure are of importance for interpreting the optical properties. For example a grading of the refractive index as well as optical anisotropy may occur, and SE enables us to detect details of such changes. In addition, thickness nonuniformities of the films can be modeled.

\section{Sample preparation and characterization}

The films were prepared with dual-target reactive DC magnetron sputtering using a deposition system based on a Balzers Model UTT 400 unit. The targets were 5-cm-diameter metallic plates of $\mathrm{W}$ and Ni having $99.9 \%$ purity. Sputtering was carried out under a constant 
$\mathrm{O}_{2} /$ Ar gas-flow ratio of 0.15 , which is optimal for sputtering of pure tungsten oxide [6]. The working pressure was $\sim 30$ mTorr. Ar and $\mathrm{O}_{2}$ were both of $99.998 \%$ purity. The total discharge power was set to $230 \mathrm{~W}$, and the power delivered to the individual targets was varied in order to obtain various compositions of $\mathrm{Ni}_{x} \mathrm{~W}_{1-x}$ oxide thin films. Films of all compositions were deposited on silicon (111) substrates at room temperature. A detailed description of the sample preparations and determination of compositions of the $\mathrm{Ni}_{x} \mathrm{~W}_{1-x}$ oxides is given elsewhere [6].

The structure of the films was determined by X-ray diffraction (XRD) using a Siemens Model D5000 diffractometer operating with $\mathrm{Cu} \mathrm{K}_{\alpha}$ radiation in a conventional $\Theta-2 \Theta$ setup. No clear XRD patterns were produced by the $\mathrm{Ni}_{\mathrm{x}} \mathrm{W}_{1-\mathrm{x}}$ oxide films; hence these films are characterized as X-ray amorphous.

Cross-sectional film morphologies were investigated by scanning electron microscopy (SEM) using a LEO Ultra 55 FEG instrument. A typical cross-sectional view on a fractured sample is shown in Fig. 1. Columnar structures of the films as well as porosity are evident from the SEM micrograph. The thicknesses of the films were found to be in the range $125<d<250 \mathrm{~nm}$.

\section{Acquisition and analysis of ellipsometry data}

Spectroscopic ellipsometry measurements were performed with a variable-angle ellipsometer (VASE from J.A. Woollam Co., Inc.) in the $0.63-6.18 \mathrm{eV}$ spectral range with a step of 0.02 eV. In addition, an infrared Fourier transform rotating compensator ellipsometer (J.A. Woollam Co., Inc.) was used in the $500-5200 \mathrm{~cm}^{-1}$ wavenumber interval with a resolution of 4 $\mathrm{cm}^{-1}$. Three to five angles of incidence were used in the $50-70^{\circ}$ range. The measured ellipsometric angles $\Psi$ and $\Delta$ are defined from the ratio of the reflection coefficients $R_{p}$ and $R_{s}$ for the $p$ and $s$ polarizations, respectively, (i.e., polarization of the electric field parallel and perpendicular to the plane of incidence) [8] according to

$$
\frac{R_{p}}{R_{s}}=\tan \Psi \exp (i \Delta)
$$

Data analysis was performed with the WVASE32 software [9]: the found solutions were evaluated according to the Levenberg-Marquardt method for minimizing the mean-squared error (MSE) defined by

$$
M S E=\frac{1}{2 N-M} \sum_{i=1}^{N}\left[\left(\frac{\Psi_{i}^{\bmod }-\Psi_{i}^{\exp }}{\sigma_{\psi, i}^{\exp }}\right)^{2}+\left(\frac{\Delta_{i}^{\bmod }-\Delta_{i}^{\exp }}{\sigma_{\Delta, i}^{\exp }}\right)^{2}\right],
$$


where $N$ is the number of $(\Psi, \Delta)$ pairs, $M$ is the number of fitted parameters in the model and $\sigma$ are standard deviations of the experimental data points. The superscripts mod and exp indicate model-generated and experimental data, respectively.

Data for the Si substrate with native oxide were first analyzed. The thickness of the $\mathrm{SiO}_{2}$ layer was determined to be $2.9 \pm 0.1 \mathrm{~nm}$ by using optical constants of $\mathrm{Si}$ and native oxide taken from the literature [10]. In the further calculations, we kept the $\mathrm{SiO}_{2}$ layer thickness constant.

The structural model used in the analysis of $\mathrm{Ni}_{x} \mathrm{~W}_{1-x}$ oxide films on $\mathrm{Si}$ can be expressed as $\mathrm{Si}$ substrate/ $\mathrm{SiO}_{2} / \mathrm{Ni}_{x} \mathrm{~W}_{1-x}$ oxide/overlayer/ambient. A thickness inhomogeneity of the $\mathrm{Ni}_{\mathrm{x}} \mathrm{W}_{1-\mathrm{x}}$ oxide layer was included in order to further improve the fit. The overlayer, representing roughness with a thickness $d_{\text {overlayer }}$, is accounted for by an effective medium approximation (EMA), which is used to represent an inhomogeneous material in terms of an

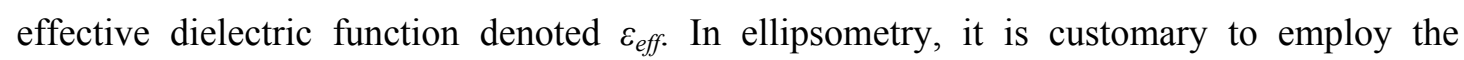
Bruggeman EMA [11] defined by

$$
\sum_{i=1}^{m} f_{i} \frac{\varepsilon_{i}-\varepsilon_{e f f}}{\varepsilon_{i}+2 \varepsilon_{e f f}}=0, \quad \sum_{i=1}^{m} f_{i}=1
$$

where $m$ is the number of components ( $m=2$, i.e. $\mathrm{Ni}_{x} \mathrm{~W}_{1-x}$ oxide and void in our case), and $f_{i}$ and $\varepsilon_{i}$ are the volume fraction and the complex dielectric function of component $i$, respectively. For each sample in the present analysis, the volume fraction of $\mathrm{Ni}_{x} \mathrm{~W}_{1-x}$ oxide and voids was taken to be 0.5 for the overlayer.

The spectral dependencies of the optical properties of the $\mathrm{Ni}_{\mathrm{x}} \mathrm{W}_{1-\mathrm{x}}$ oxides can be described in terms of their complex dielectric function $\varepsilon(E)=\varepsilon_{1}(E)+i \varepsilon_{2}(E)$ or complex refractive index $N=n+i k=\sqrt{\varepsilon(E)}$, where $E$ is the photon energy. A parametric model was used for $\varepsilon$, and several oscillator models were used as specified next.

We first consider models for the optical properties at short wavelengths in the region of the fundamental band gap. In this case a Lorentz $(L)$ oscillator was used as one of the options. In this model the contribution $\varepsilon_{L}$ to $\varepsilon$ is given by [8]

$$
\varepsilon_{L}=\frac{A \Gamma E_{0}}{E_{0}^{2}-E^{2}-i \Gamma E},
$$

where $\varepsilon, A$ is the amplitude, $\Gamma$ is a broadening parameter, and $E_{0}$ is the resonance energy.

A Tauc-Lorentz (TL) oscillator represents another choice. A Tauc expression for the imaginary part of the dielectric function near the band edge is then employed in combination 
with a Lorentz oscillator [12] to define $\varepsilon_{T L}$. Using this model for the absorption onset region at photon energies slightly greater than the Tauc gap $E_{g}$, one obtains $\varepsilon_{2 T L}(E) \infty\left[\left(E-E_{g}\right)^{2} / E^{2}\right]$, assuming a constant momentum matrix element and parabolic bands. Considering only a single transition, $\varepsilon_{2 T L}$ of the TL model is then given by [8]

$$
\varepsilon_{2 T L}=\frac{A E_{0} \Gamma\left(E-E_{g}\right)^{2}}{\left(E^{2}-E_{0}^{2}\right)^{2}+E^{2} \Gamma^{2}} \frac{\Theta\left(E-E_{g}\right)}{E}
$$

where $\Theta\left(E-E_{g}\right)$ is the Heaviside function $\left[\Theta\left(E-E_{g}\right)=1\right.$ for $E \geq E_{g}$ and $\Theta\left(E-E_{g}\right)=0$ for $E<$ $E_{g}$ ]. The expression for $\varepsilon_{1 T L}$ is obtained via a Kramers-Kronig integration of $\varepsilon_{2 T L}$ [8].

We now turn to expressions for the dielectric function in the infrared region (IR). Here we could use models based on Gaussian absorption profiles. In the Gauss $(G)$ oscillator model, $\varepsilon_{2 G}$ is given by [9]

$$
\varepsilon_{2 G}=A e^{-\left(\frac{E-E_{0}}{\sigma}\right)^{2}}-A e^{-\left(\frac{E+E_{0}}{\sigma}\right)^{2}}
$$

where $\sigma$ is a broadening parameter and $\varepsilon_{1 G}$ is obtained via a Kramers-Kronig integration. In the Gaussian model the full width of the lineshape at half maximum is given by $\Gamma=2 \sqrt{\ln (2)} \sigma$.

In summary the used model dielectric function is

$$
\varepsilon=\varepsilon_{\infty}+\varepsilon_{T L}+\sum_{i} \varepsilon_{G i}
$$

$\varepsilon_{\infty}$ is the high-frequency contribution. However, for the $\mathrm{Ni}_{x} \mathrm{~W}_{1-x}$ oxide film with $x=0.90, \varepsilon_{T L}$ was replaced with $\varepsilon_{L}$.

\section{Results and discussion}

Five samples of $\mathrm{Ni}_{x} \mathrm{~W}_{1-x}$ oxide, with $x$ equal to $0,0.05,0.19,0.43$ and 0.90 were studied. Figure 2 shows, as an example, experimental and fitted model data on $\Psi$ and $\Delta$ for a $\mathrm{Ni}_{0.19} \mathrm{~W}_{0.81}$ oxide film on $\mathrm{Si}$.

The best fit parameter values from the parametric models used to describe the dielectric functions of amorphous $\mathrm{Ni}_{x} \mathrm{~W}_{1-x}$ oxides with different compositions are summarized in Tables 1 and 2. The non-uniformity of the film thicknesses is around 3-5\% of the average 
thickness. The MSE values in our calculations were of the order of 4 or less, which corresponds to relatively good fittings. Table 1 shows parameter values for the $T L$ oscillator describing the resonance within the ultraviolet (UV) spectral region and the Gauss oscillator located near $0.4 \mathrm{eV}$. These oscillators are present for $\mathrm{Ni}_{x} \mathrm{~W}_{1-x}$ oxides with $x$ varying from 0 to 0.43 . Table 2 summarizes the parameter values of the oscillators describing absorption in the infrared (IR) region from $0.062 \mathrm{eV}$ to $0.165 \mathrm{eV}$. The dielectric functions of $\mathrm{Ni}_{x} \mathrm{~W}_{1-x}$ oxides for these wavelengths can be represented by a set of Gauss oscillators (cf., Table 2), because increasing the content of $\mathrm{Ni}$ leads to the formation of new oxide phases. For the oxide with $x=0.90$ a single Gauss oscillator was found to be sufficient. The dispersion of the refractive index and extinction coefficient of $\mathrm{Ni}_{x} \mathrm{~W}_{1-x}$ oxides at different compositions are shown in Figs. 3 and 4.

From Table 1 it is evident that the bandgap of the mixed metal oxide films is between $3.2 \mathrm{eV}$ and $3.3 \mathrm{eV}$ for all films with $x$ up to 0.43 , and close to the bandgap of amorphous $\mathrm{WO}_{3}$ [1]. These mixed oxide films have larger resonance energies and broader $T L$ absorption bands than for pure tungsten oxide [5]. The broadening effect may originate from the larger amount of defects in the two-component material. It should be noted, that $\mathrm{Ni}_{0.90} \mathrm{~W}_{0.10}$ oxide absorbed in the visible range whereas the other oxides were transparent. The absorption in the visible may be because the nickel oxide contains $\mathrm{Ni}^{3+}$ which exists in the phases $\mathrm{NiOOH}$ and/or $\mathrm{Ni}_{2} \mathrm{O}_{3}[13]$.

Interband absorption in $\mathrm{WO}_{3}$ seems to be very dependent on the crystalline structure of the material, as well as on the stoichiometry, and it is difficult to find data for the position of the resonance energy $\left(E_{0}\right)$ for amorphous thin films. However, DeVries et al. [14] have studied sputter deposited thin films and found a peak in the imaginary part of the dielectric function at $4.5 \mathrm{eV}$, in agreement with our data. On the other hand no such peak was seen for evaporated films up to $4.8 \mathrm{eV}$ [15]. For $\mathrm{NiWO}_{4}$ the maximum appears to be at higher energies in the vicinity of $6 \mathrm{eV}$ [16]. The shift of $E_{0}$ towards higher energies as $x$ increases as observed in Table 1 would then be consistent with an enhanced amount of $\mathrm{NiWO}_{4}$ in the films, in agreement with the conclusions in our earlier investigation [6]. The Ni-rich film with $x=0.9$ exhibits a lower imaginary part of the dielectric function in the UV region. This film exhibits a broad peak in the $4 \mathrm{eV}$ to $4.5 \mathrm{eV}$ region, which is consistent with the interband absorption in $\mathrm{NiO}[17]$.

In the visible range, the values of $n$ of the studied mixed oxides were very close to $n$ of pure tungsten oxide and smoothly decreased for increasing amounts of Ni (see Fig. 3). This behaviour of $n$ can be caused by stoichiometric or structural variations. 
The origin of the Gauss oscillator at $0.4 \mathrm{eV}$ is unknown, although broad mid-IR absorption at somewhat different energies has been observed previously in $\mathrm{WO}_{3}[14,18]$. This absorption could be associated with gap states or possibly related to polaronic transitions. The IR absorption in $\mathrm{WO}_{3}$ is dominated by a strong peak close to $0.08 \mathrm{eV}$, due to the $\mathrm{O}-\mathrm{W}-\mathrm{O}$ stretching mode $[14,15,18]$. In addition to this we also observe a subsidiary peak at $0.09 \mathrm{eV}$ for films with $\mathrm{x}<0.1$, as seen in Fig. 4. This peak is usually interpreted as due to $\mathrm{W}=\mathrm{O}$ vibrations [6] and its presence points to some degree of sub-stoichiometry in our tungsten oxide films.

The IR absorption for films with higher magnitudes of $x$ seems to be dominated by these vibrations, although the positions differ somewhat. In the case of films with high $x$, the $\mathrm{W}=\mathrm{O}$ vibration, which decreases with increasing $\mathrm{Ni}$ content as expected, seems to be most prominent. No features due to $\mathrm{NiO}$ can be identified probably due to the low energy cutoff in the measurement.

\section{Concluding remarks}

A set of thin films of $\mathrm{Ni}_{x} \mathrm{~W}_{1-x}$ oxide with different compositions $x$ and grown on Si were characterized by XRD, SEM and SE. All two-component materials were amorphous and exhibited columnar structures. Four-phase models with homogenous $\mathrm{Ni}_{x} \mathrm{~W}_{1-x}$ oxide layers were applied to analyze the SE data. Parametric models were used to determine film and overlayer thicknesses, thickness non-uniformity, and optical functions in the spectral range from 0.062 to $5.905 \mathrm{eV}$ for different compositions. The results were interpreted by comparison with known optical properties of $\mathrm{WO}_{3}, \mathrm{NiO}$ and $\mathrm{NiWO}_{4}$. As far as we know no accurate optical constants have previously been reported for mixed nickel tungsten oxides.

\section{Acknowledgements}

This work was supported by a grant from the Swedish Research Council for Environment, Agricultural Sciences and Spatial Planning (FORMAS). The Knut and Alice Wallenberg Foundation is acknowledged for financial support to instrumentation.

\section{References}

[1] C.G. Granqvist, Handbook of Inorganic Electrochromic Materials, Elsevier, Amsterdam, The Netherlands, 1995.

[2] G.A. Niklasson, C.G. Granqvist, J. Mater. Chem. 17 (2007) 127. 
[3] C.G. Granqvist, Solar Energy Mater. Solar Cells 91 (2007) 1529.

[4] S. Green, J. Backholm, P. Georén, C.G. Granqvist, G.A. Niklasson, Solar Energy Mater. Solar Cells 93 (2009) 2050.

[5] I. Valyukh, S. Green, H. Arwin, G.A. Niklasson, E. Wäckelgård, C.G. Granqvist, Solar Energy Mater. Solar Cells 94 (2010) 724.

[6] S.V. Green, A. Kuzmin, J. Purans, C.G. Granqvist, G.A. Niklasson, unpublished.

[7] C.G. Granqvist, S. Green, G.A. Niklasson, N.R. Mlyuka, S. von Kræmer, P. Georén, Thin Solid Films 518 (2010) 3046.

[8] H.G. Tompkins, E.A. Irene (Eds), Handbook of Ellipsometry, William Andrew Publishing, New York, USA, 2005.

[9] J.A. Woollam Co., Inc, Guide to using WVASE32, WexTech Systems.

[10] C.M Herzinger, B. Johs, W.A. McGahan, J.A. Woollam, W. Paulson, J. Appl. Phys. 83 (1998) 3323.

[11] D.A.G. Bruggeman, Ann. Phys., 5th Series (Leipzig) 24 (1935) 636.

[12] J. Tauc, R. Grigorovici, A. Vancu, phys. stat. sol. (b) 15 (1966) 627.

[13] E. Avendaño, H. Rensmo, A. Azens, A. Sandell, G. de M Azevedo, H. Siegbahn, G.A. Niklasson, C.G. Granqvist, J. Electrochem. Soc. 156 (2009) P132.

[14] M.J. DeVries, C. Trimble, T.E. Tiwald, D.W. Thompson, J.A. Woollam, J. Vac. Sci. Technol. A 17 (1999) 2906.

[15] E. Pasqual, J. Marti, E. Garcia, A. Canillas, E. Bertran, Thin Solid Films 313-314 (1998) 682.

[16] T. Ejima, T. Banse, H. Takatsuka, Y. Kondo, M. Ishino, N. Kimura, M. Watanabe, I. Matsubara, J. Luminesc. 119-120, (2006) 59.

[17] R.J. Powell, W.E. Spicer, Phys. Rev. B 2 (1970) 2182.

[18] E.B. Franke, C.L. Trimble, J.S. Hale, M. Schubert, J.A. Woollam, J. Appl. Phys. 88 (2000) 5777. 


\section{List of figure and table captions}

Figure 1. SEM cross-section of a $\mathrm{Ni}_{x} \mathrm{~W}_{1-x}$ oxide film with $\mathrm{x}=0.19$. The scale bar indicates $100 \mathrm{~nm}$.

Figure 2. Spectral ellipsometric data $\Psi$ and $\Delta$ at angles of incidence from $50^{\circ}$ to $70^{\circ}$ for a $\mathrm{Ni}_{0.19} \mathrm{~W}_{0.81}$ oxide film on $\mathrm{Si}$ within the range a) from $0 \mathrm{eV}$ to $6 \mathrm{eV}$; b) from $0 \mathrm{eV}$ to $1 \mathrm{eV}$. The experimental results are indicated by dashed curves, and the data fitted to a model discussed in the text are shown by solid curves.

Figure 3. Dependencies of refractive index $n$ for $\mathrm{Ni}_{\mathrm{x}} \mathrm{W}_{1-\mathrm{x}}$ oxides versus photon energy within the range a) from $0 \mathrm{eV}$ to $6 \mathrm{eV}$; b) from $0.05 \mathrm{eV}$ to $0.50 \mathrm{eV}$.

Figure 4. Dependencies of the extinction coefficient $k$ for $\mathrm{Ni}_{\mathrm{x}} \mathrm{W}_{1-\mathrm{x}}$ oxides versus photon energy within the range a) from $0 \mathrm{eV}$ to $6 \mathrm{eV}$; b) from $0.05 \mathrm{eV}$ to $0.50 \mathrm{eV}$.

Table 1. Best fit parameter values obtained from models for $\mathrm{Ni}_{x} \mathrm{~W}_{1-x}$ oxide films with different compositions $x$ in the spectral range $0.165-5.905 \mathrm{eV}$. The $90 \%$ confidence limits are better than \pm 0.01 except for thicknesses $( \pm 0.1)$ and amplitudes of Tauc-Lorentz and Lorentz oscillators ( \pm 0.3$)$.

Table 2. Best fit parameter values obtained from models for $\mathrm{Ni}_{x} \mathrm{~W}_{1-x}$ oxide films with different compositions $x$ in the spectral range $0.062-0.165 \mathrm{eV}$. The values for the $90 \%$ confidence limits are better than \pm 0.01 for all parameters. 


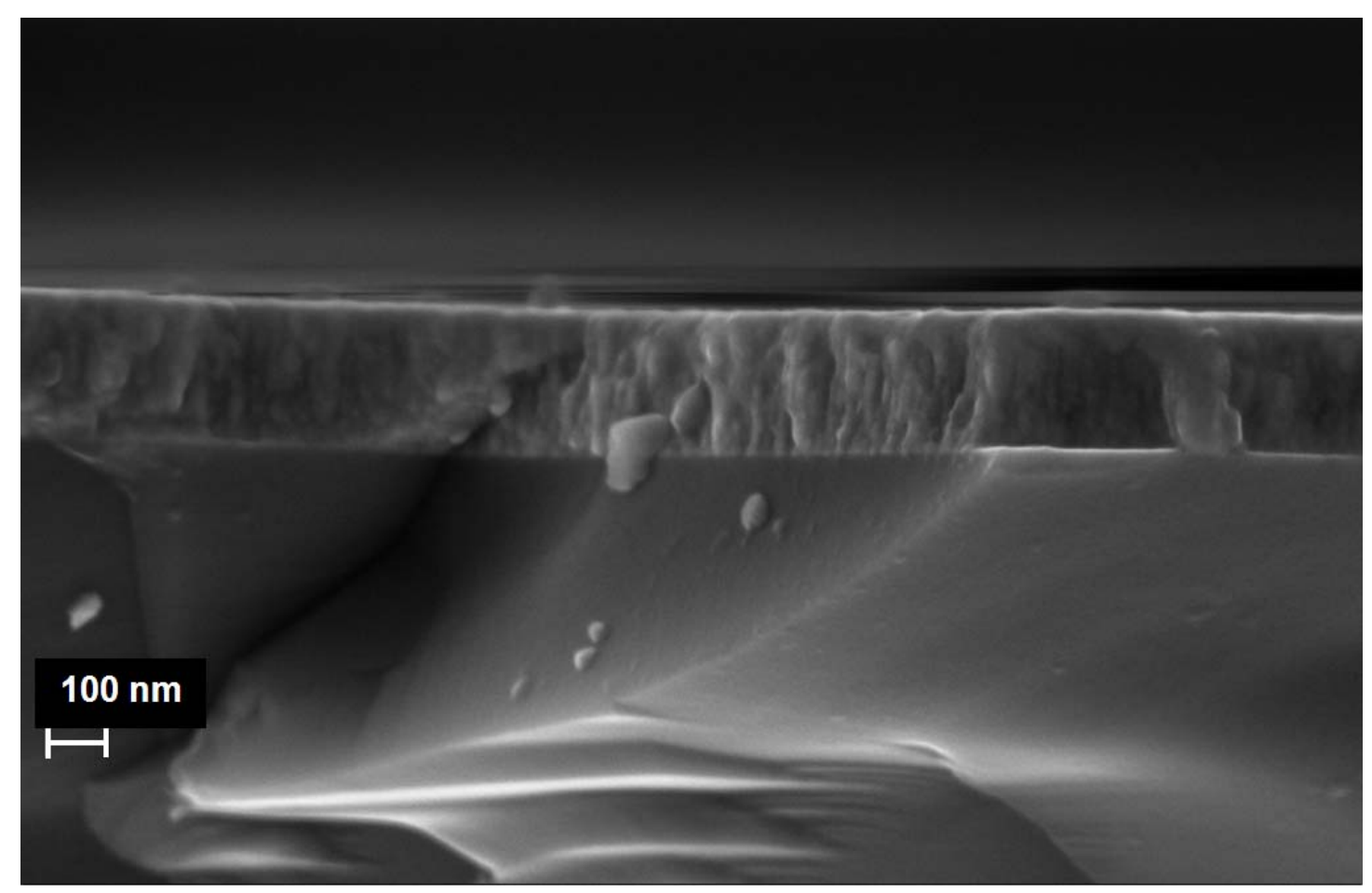

Figure 1. 

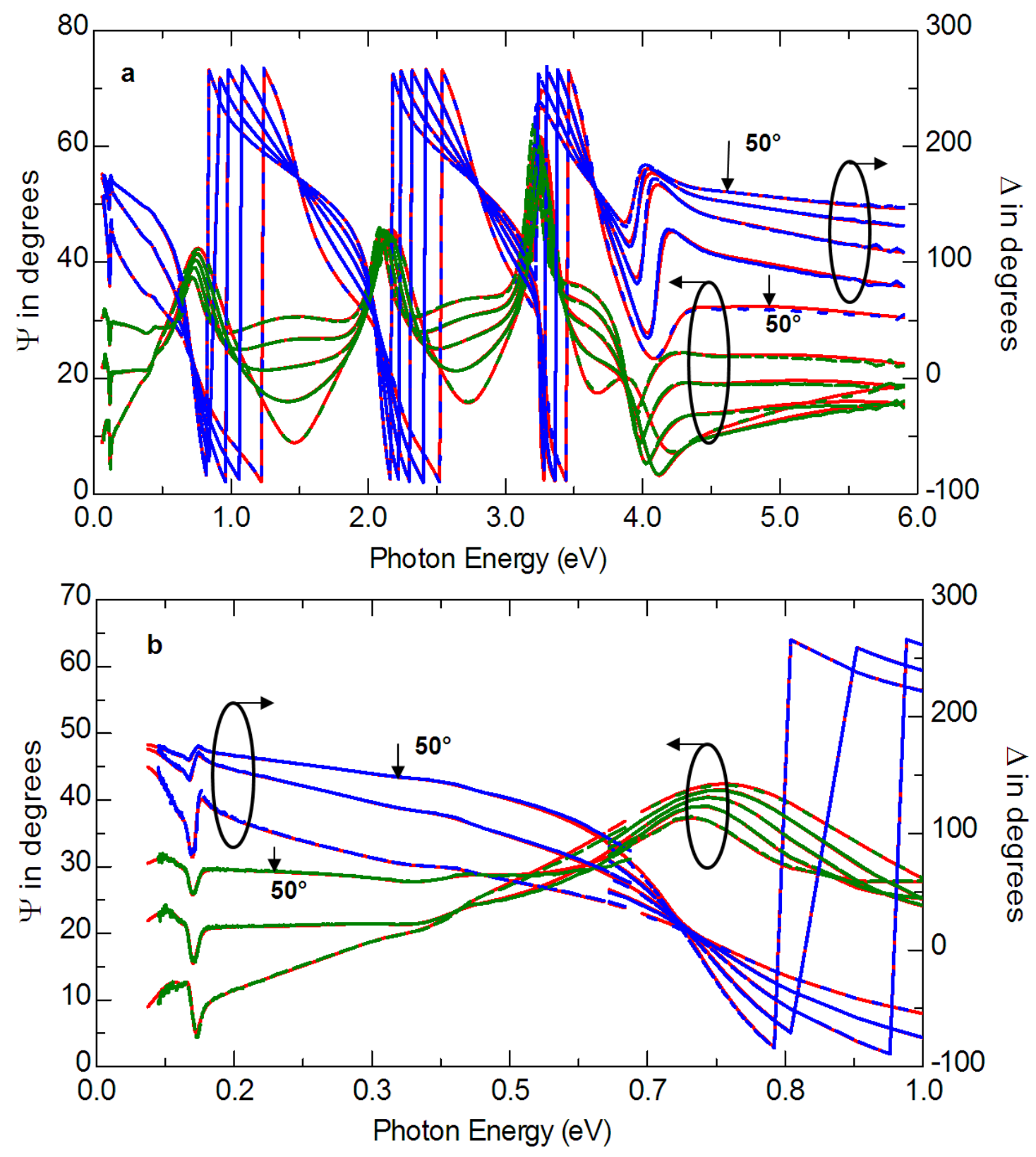

Figure 2. 

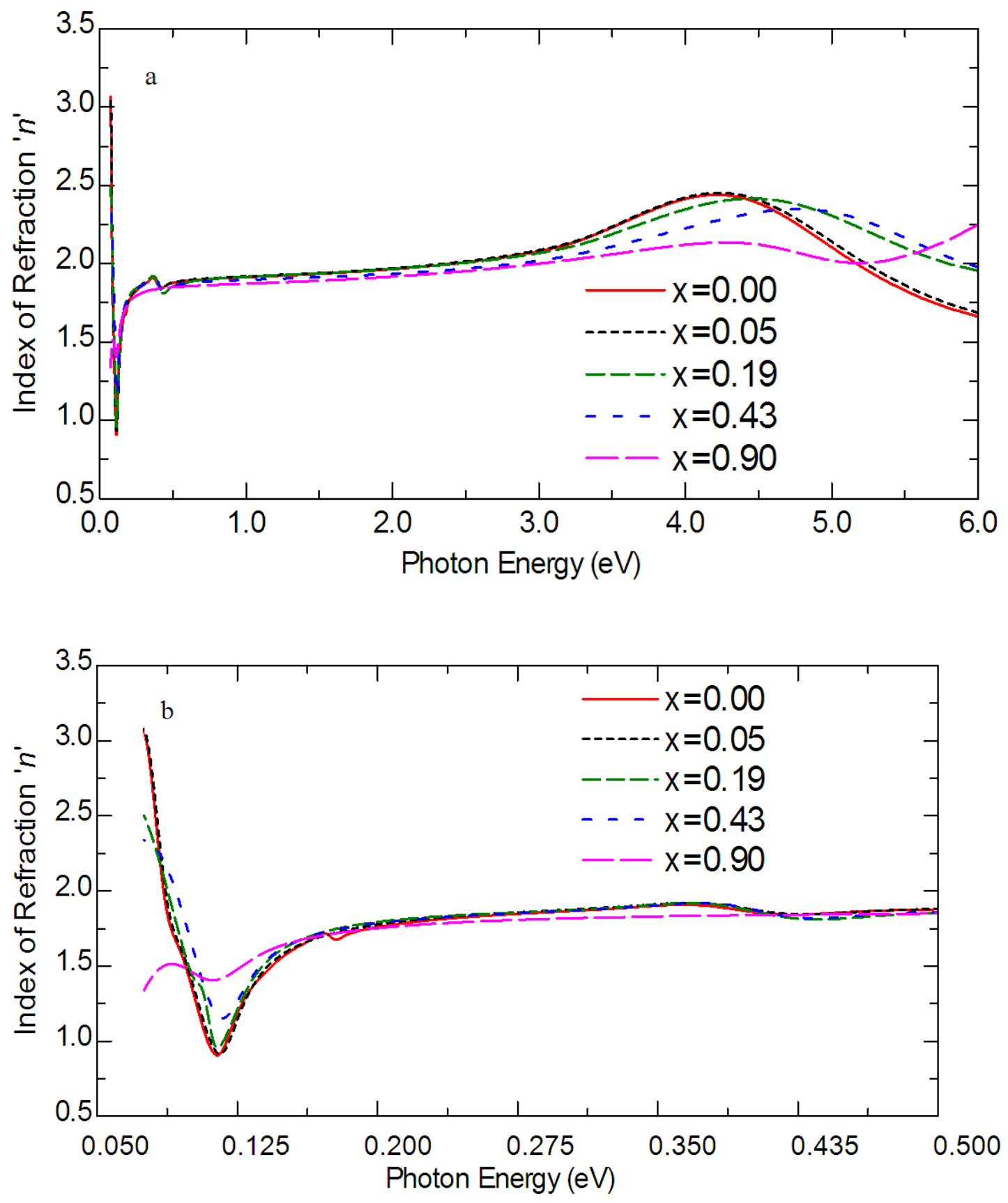

Figure 3. 

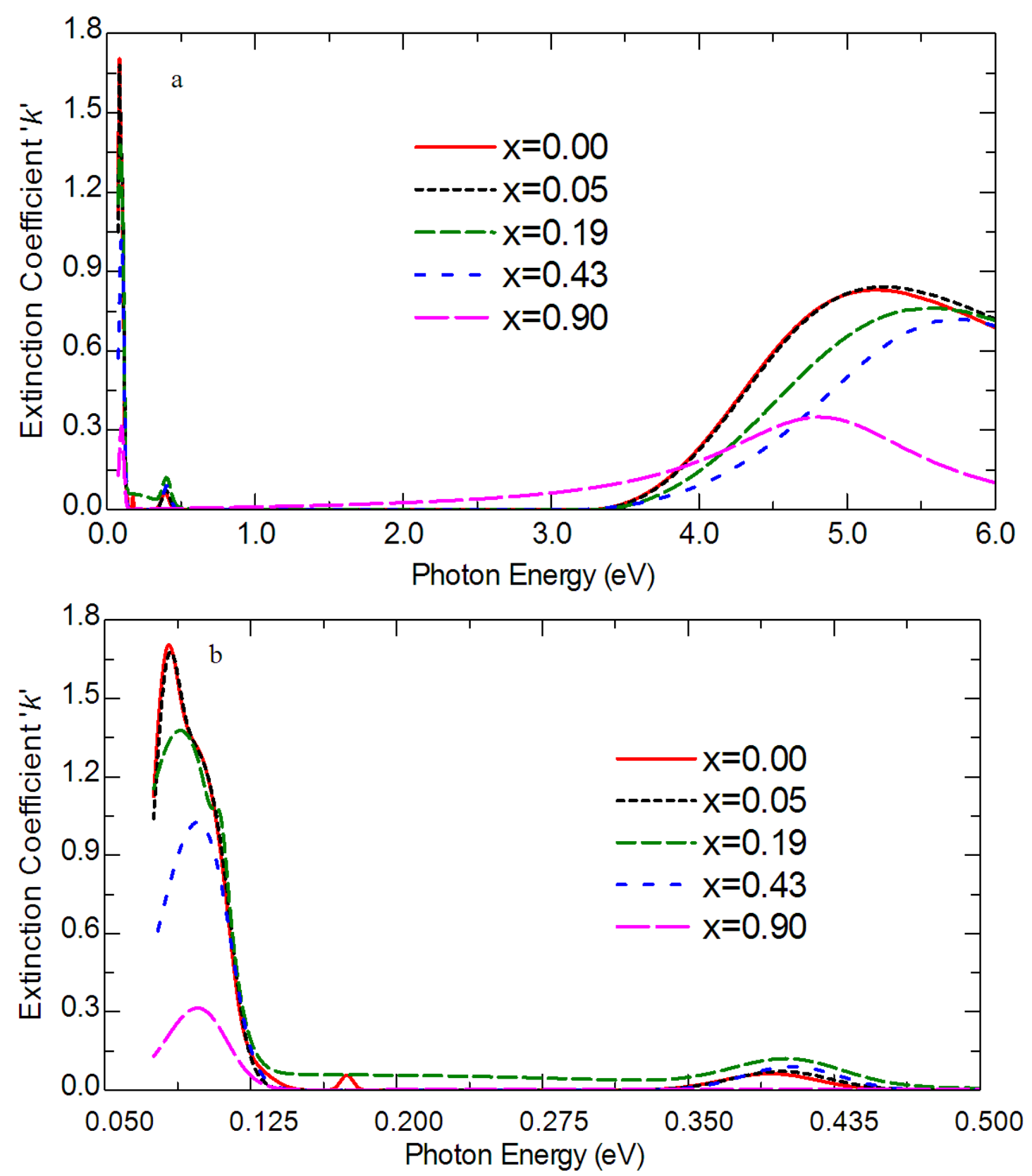

Figure 4. 
Table 1.

\begin{tabular}{|c|c|c|c|c|c|c|c|c|c|c|}
\hline \multirow[t]{2}{*}{$x$} & \multirow{2}{*}{$\begin{array}{c}d \\
(\mathrm{~nm})\end{array}$} & \multirow{2}{*}{$\begin{array}{c}d_{\text {overlayer }} \\
(\mathrm{nm})\end{array}$} & \multirow[t]{2}{*}{$\varepsilon_{\infty}$} & \multicolumn{4}{|c|}{ TL oscillator } & \multicolumn{3}{|c|}{ Gauss oscillator } \\
\hline & & & & $\begin{array}{l}A \\
(*)\end{array}$ & $\begin{array}{c}E_{0} \\
(\mathrm{eV})\end{array}$ & $\begin{array}{c}\Gamma \\
(\mathrm{eV})\end{array}$ & $\begin{array}{c}E_{g} \\
(\mathrm{eV})\end{array}$ & $\begin{array}{l}A \\
(*)\end{array}$ & $\begin{array}{c}E_{0} \\
(\mathrm{eV})\end{array}$ & $\begin{array}{c}\Gamma \\
(\mathrm{eV})\end{array}$ \\
\hline 0.00 & 247.9 & 11.1 & 1.00 & 75.3 & 4.52 & 2.08 & 3.24 & 0.24 & 0.39 & 0.06 \\
\hline 0.05 & 266.7 & 7.4 & 1.00 & 79.3 & 4.55 & 2.16 & 3.26 & 0.27 & 0.40 & 0.06 \\
\hline 0.19 & 240.2 & 6.2 & 1.88 & 69.7 & 4.89 & 2.67 & 3.27 & 0.37 & 0.40 & 0.07 \\
\hline 0.43 & 207.2 & 5.0 & 2.10 & 40.5 & 5.35 & 2.44 & 3.11 & 0.34 & 0.40 & 0.06 \\
\hline $0.90^{\#}$ & 124.5 & 6.4 & 1.29 & 1.43 & 4.84 & 1.69 & - & - & - & - \\
\hline
\end{tabular}

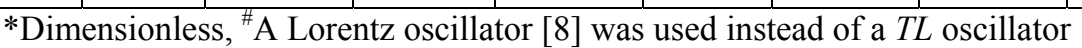


Table 2.

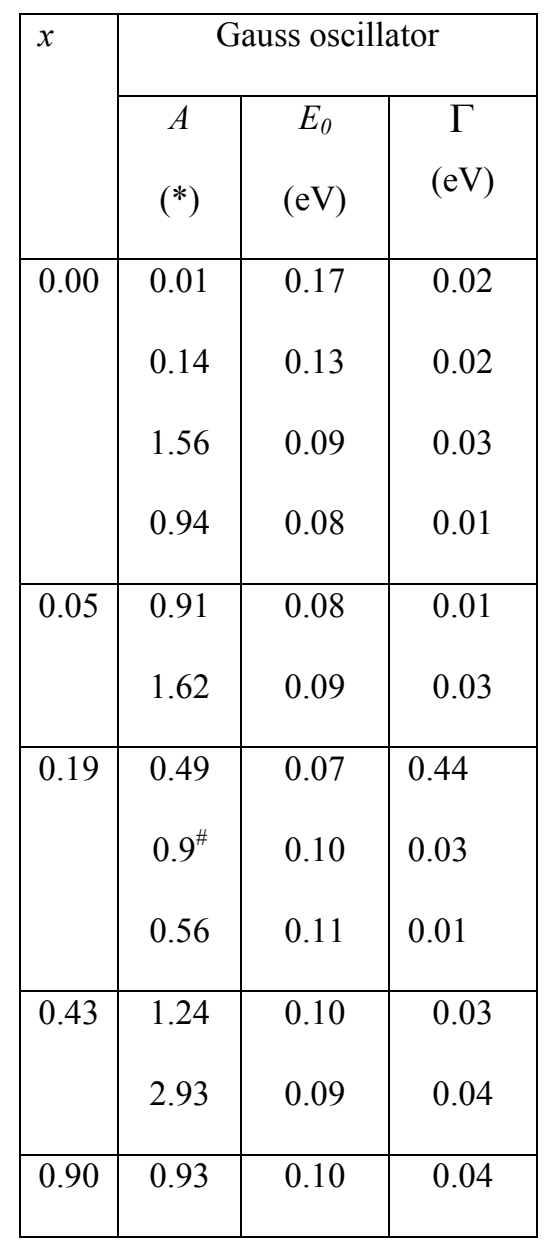

*Dimensionless, ${ }^{\#}$ The confidence limit is \pm 0.3 . 